\title{
A new method for testing wide range horizontal field angle
}

\author{
Sijie Huang*, Jin Huang, Shujie Wang, Zhenwei Ma, and Shangyu Gu \\ The Third Research Institute of the Ministry of Public Security, Shanghai, 200033, China
}

\begin{abstract}
In order to solve the problems of small measurement range, large error and low efficiency of laboratory optical field angle testing, a high-precision, easy -operating, high-efficient, and widely used horizontal field angle test method is proposed. It comes to a conclusion that the test method can reduce the experimental error through the analysis of the principle of the field of view error and the calculation of laboratory simulation. The simulation results show that for cameras with a field of view of more than 150 degrees, the measurement error can be reduced by 37 degrees, and when the field of view of the camera under test is close to 170 degrees, the method can reduce the measurement error by nearly 54 degrees. Meanwhile, a wide-range horizontal field angle measurement method is proposed. The camera under test is moved on the supporting mobile platform to image the target test board, and then the imaging target is read by reading the scale value on the test board calculates the angle of the camera under test. This method can effectively avoid the measurement error of the angle caused by the distance between the center of the lens surface and the center of the entrance pupil, so as to quickly obtain the angle of view test results, and improve the testing accuracy, and it is also suitable for cameras that measure a wide range of field angles (wide-angle camera or fisheye camera, etc.) to solve the problem of laboratory testing a wide range of horizontal field angles.
\end{abstract}

Keywords: Field of view, Surveillance camera, Wide range, Test accuracy.

\section{Introduction}

The optical system is one of the most important components of an imaging camera. The camera's field of view angle is an important indicator of the camera's optical system, which characterizes the camera's imaging angle range. Therefore, the test of the camera's field of view has become an important part of evaluating the performance of a video surveillance camera.

Currently, there are many methods for measuring the optical field of view of the imaging system. Jiang Lili et al. proposed a method using a transmissive film test card with several concentric circles and digital scales in the background. The field of view angle can

\footnotetext{
*Corresponding author: huangsijie111@126.com
} 
be measured by reading the scales. test ${ }^{[1]}$. Different from the former, Yuan Li's method is more intuitive. He proposed installing a camera the on a turntable to image the star point hole placed at the focal plane of the collimator, when rotating the turntable, the camera can image the star point hole from the extreme edge of one end. to the other end ${ }^{[2]}$. By this way, the angle of rotation of the turntable is the angle of view of the camera. And the same method can be used to measure the angle of view in another direction by rotating the camera by 90 degrees.

The proposed methods can be performed as formula (1):

$$
\tan \alpha=\frac{W}{2 L}
$$

On the basis of the above method, Li Kun et al. proposed a method and device for camera field of view calibration. The camera's field of view angle is obtained by the distance of the camera moving along the optical axis $L_{1}-L_{2}$ and the width difference of the corresponding field of view $W_{1}-W_{2}{ }^{[3]}$, as follows

$$
\tan \alpha=\frac{W}{2 L_{1}}=\frac{W}{2 L_{2}}=\frac{W_{1}-W_{2}}{2\left(L_{1}-L_{2}\right)}
$$

LI et al. of the China Air-to-Air Missile Research Institute have designed a semiconductor laser fuze field angle measurement device ${ }^{[4]}$, which mainly includes a mirror turntable and a one-dimensional translation stage; TAN et al. of Huazhong Agricultural University designed an automatic test system for the field of view of the laser fuze receiving optical system ${ }^{\left[{ }^{]}\right]}$; FENG et al. of Harbin Institute of Technology proposed a method of measuring the field of view angle of laser guidance by using a three-axis turntable $^{[6]}$; Yang Bowen et al. designed the infrared light-emitting diode with adaptive brightness as the target point, and used the displacement of the coordinate measuring machine to construct a virtual three-dimensional target ${ }^{[7]}$; Lin Wukang researched a large field of view calibration method based on improved flexible plane targets, small target splicing, and auxiliary targets. ${ }^{[8]}$; Peng et al. proposed an internal parameter calibration method based on a one-dimensional target ${ }^{[9]}$, Gao Ting improved the concentric circle detection algorithm and verified it through experiments ${ }^{\left[10^{]}\right.}$; On the basis of previous research methods, Li Weimin et al. proposed a method for calibration of a differential large field of view camera ${ }^{[11,12]}$; Ye Dong et al. proposed a camera parameter calibration method based on the minimum distance from the calibration control point of the virtual stereo target to the corresponding anti-projection line ${ }^{[13]}$, In addition, genetic algorithm is applied to the camera parameter calibration method based on virtual stereo target ${ }^{[14]}$; Sun Junhua et al. proposed that by placing multiple smaller two-dimensional targets in the calibration space, the fixed relative positions between the small targets were used to establish the connection between the targets, so that the multiple targets were distributed in the entire calibration space, so as to control the camera Parameter calibration ${ }^{[15]}$; An Yan et al. proposed a device for receiving the angle of view of portable laser communication. By fixing the autocollimator and the laser communication machine on the mounting plate, and adjusting the azimuth and tilting table to achieve small-scale high-precision testing ${ }^{[16,17]}$.

The operation steps of the above methods are relatively complicated and are not suitable for high-efficiency laboratory test operations. For cameras with a wide field of view, the general laboratory environment cannot be quickly and conveniently obtained due to the 
limitation of the size of the field. Greatly affect the efficiency of the test. Based on this, this article proposes a method to achieve a wide range of horizontal field of view angle testing, simulation and error analysis of the field of view, and based on the analysis results designed a range of horizontal field of view device, this method can effectively avoid the lens The field angle measurement error caused by the distance between the center of the surface and the center of the entrance pupil improves the test accuracy.

\section{Imaging principle of photoelectric imaging system}

A typical photoelectric imaging system is mainly composed of image sensor, optical lens, signal transmission control processing module and display storage module, as shown in figure. 1. The radiation brightness of the target scene enters the optical lens and converges to the sensor. The optical signal received by the sensor is converted into electrical signal, and the digital image is generated and processed on the display storage device.

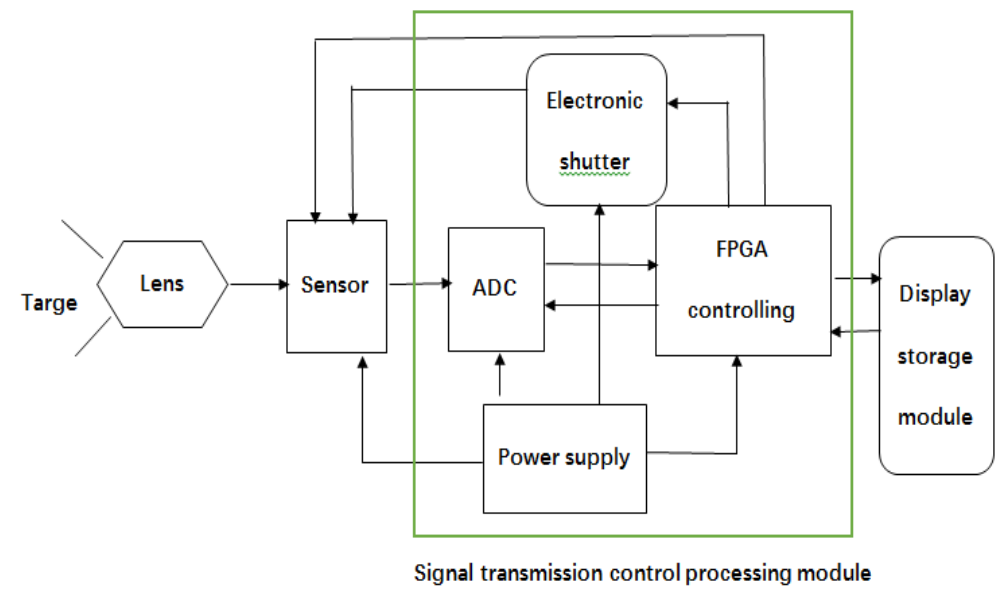

Fig. 1. Imaging system architecture diagram.

For limited distance of the object in the process of imaging, the camera's field of view angle is defined as a view angle of the pupil center line [3], the general view of the camera, because the camera surface center and into the center of the pupil distance L0 small (much smaller than L), so the camera's field of view angle of view of the camera lens approximation for the line before the opening angle of surface center. As shown in figure.2, after the line field $\mathrm{D}$ is measured with a scale, the distance $\mathrm{L}$ between the standard scale and the center of the front surface of the lens is measured, and then the imaging field angle can be calculated by formula (3)

$$
\theta=2 \arctan \left(\frac{D}{L}\right)
$$




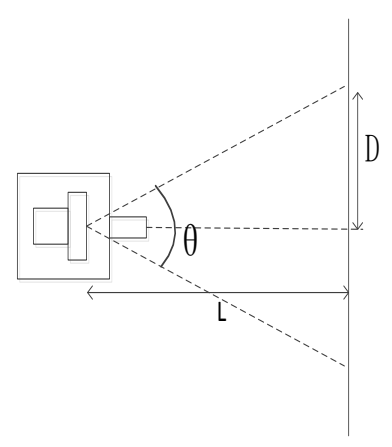

Fig. 2. Camera field of view angle (approximate) test chart.

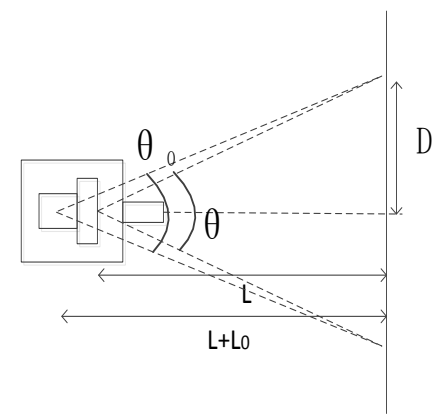

Fig. 3. Camera field of view angle (actual) test chart.

However, in actual measurement, there is a distance L0 between the lens surface center and the entry pupil center. For a camera with a relatively wide field angle, L0 will cause an error $\hat{\theta}$ in the calculated field angle. As shown in figure. 3, it is expressed by Formula (4) as follows:

$$
\hat{\theta}=\theta-\theta_{0}=2\left(\arctan \frac{D}{L}-\arctan \frac{D}{L+L_{0}}\right)
$$

In this paper, the simulation method is used to calculate and analyze the influence of the distance L0 between the lens surface center and the entry pupil center on the measurement of the camera's field angle, and a new wide range horizontal field angle test method is proposed. This method can effectively avoid the influence of L0 on the measurement of the camera's field angle, and improve the testing accuracy. The wide horizontal field angle can be measured with high efficiency in laboratory.

\section{Test error calculation simulation}

For an imaging system, the size of the field to be measured is determined by the focal length of the lens, the distance between the focal plane and the object to be photographed, and the imaging size of the object to be measured in the focal plane of the sensor, as shown in Formula (5). Where, $\mathrm{f}$ is the focal length of the lens, $\mathrm{W}$ is the imaging width of the object being photographed on the sensor target plane, $\mathrm{H}$ is the imaging height of the object being photographed on the sensor target plane, $\mathrm{L}$ is the distance from the center of the lens entering pupil to the object being photographed, $\mathrm{W}$ is the width of the object being photographed, and $\mathrm{H}$ is the height of the object being photographed. 


$$
f=w L / W=h L / H
$$

According to the application scenarios tested in the laboratory, it is assumed that the target surface size of the camera sensor is $1 / 2$ inch, the width and height are about $6.4 \mathrm{~mm} \times 4.8 \mathrm{~mm}$, the minimum focal length is $5 \mathrm{~mm}$, the field width is $1.6 \mathrm{~m}$, and the height is $1.2 \mathrm{~m}$. The distance $\mathrm{L}$ between the center of the lens entering pupil and the object being photographed can be obtained as $1.5 \mathrm{~m}$. Further, the field angle can be calculated as 65.2 degrees by formula (5). For the imaging of the scene view angle measurement, assuming that the camera lens before the surface and into the center of the pupil distance H0 change from $0.5 \mathrm{~cm}$ to $5 \mathrm{~cm}$, can be obtained by computer simulation analysis software, different $\mathrm{H} 0$ values corresponding to different camera viewing angle as a result, as shown in figure. 4 , the viewing angle measurement error between 0.2 to 2.05 degrees, The results are shown in Table 1.

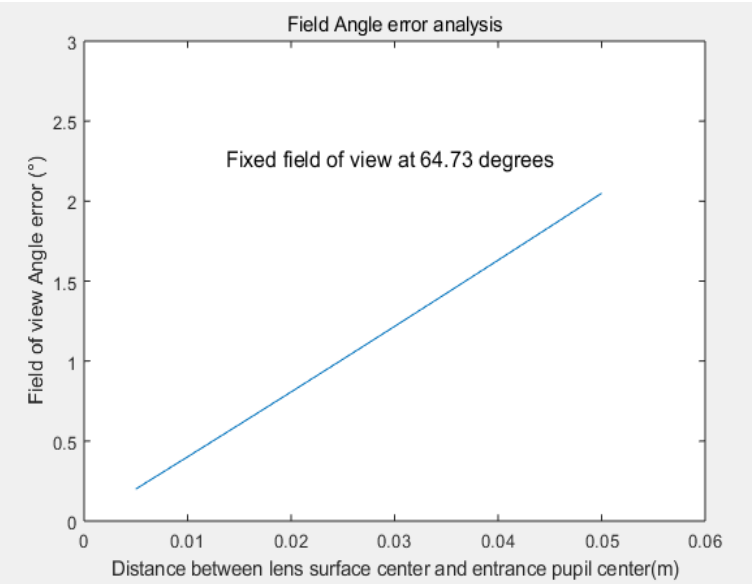

Fig. 4. Variation of field angle error with distance between lens surface center and entrance pupil center.

Table 1. The result of the change of FOV error with H0.

\begin{tabular}{|l|l|l|l|l|l|l|l|l|l|l|}
\hline H0(m) & 0.005 & 0.010 & 0.015 & 0.020 & 0.025 & 0.030 & 0.035 & 0.040 & 0.045 & 0.050 \\
\hline $\begin{array}{l}\text { Field } \\
\text { Angle } \\
\text { test } \\
\text { results }\left(^{\circ}\right)\end{array}$ & 64.93 & 65.13 & 65.34 & 65.54 & 65.74 & 65.95 & 66.16 & 66.36 & 66.57 & 66.78 \\
\hline $\begin{array}{l}\text { Field of } \\
\text { view } \\
\text { Angle } \\
\text { test } \\
\text { error }\left({ }^{\circ}\right)\end{array}$ & 0.20 & 0.40 & 0.61 & 0.81 & 1.01 & 1.22 & 1.43 & 1.63 & 1.84 & 2.05 \\
\hline
\end{tabular}

As the field angle range of the camera measured in the laboratory is wide, the field angle of some wide-angle cameras and fisheye cameras is often more than 130 degrees or greater, so further simulation is carried out, and the measurement error is calculated when the real field angle changes from 10 degrees to 170 degrees and $\mathrm{H} 0$ changes from $0.5 \mathrm{~cm}$ to $2 \mathrm{~cm}$. From figure.5, the overall curve of the field-of-view error changing with the real field-of-view angle can be obtained. To facilitate observation, log (Y) display is adopted. As can be seen from figure.6 (a), (b) and (c), when the field angle is less than 117 degrees, the rate of measurement error increases gently with the field angle, and the error range is 
within 12 degrees; when the field angle increases to more than 150 degrees, the measurement error increases to 37 degrees; when the field angle approaches 170 degrees, the measurement error approaches 54 degrees.

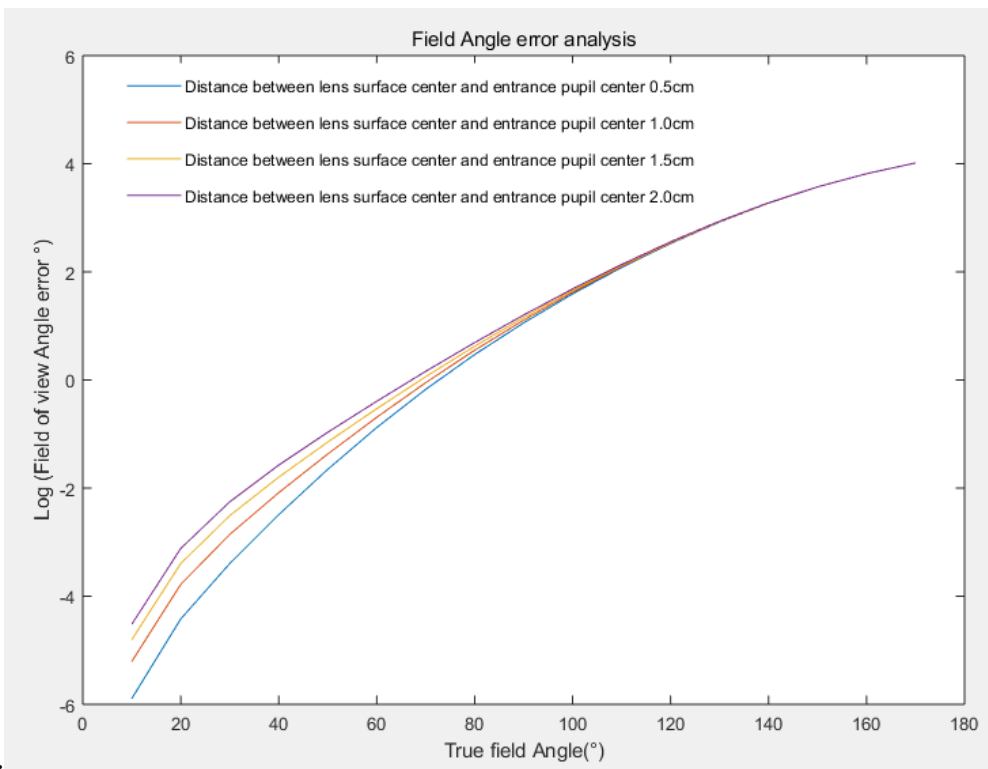

Fig. 5. Variation of global FOV angle error with real FOV angle.

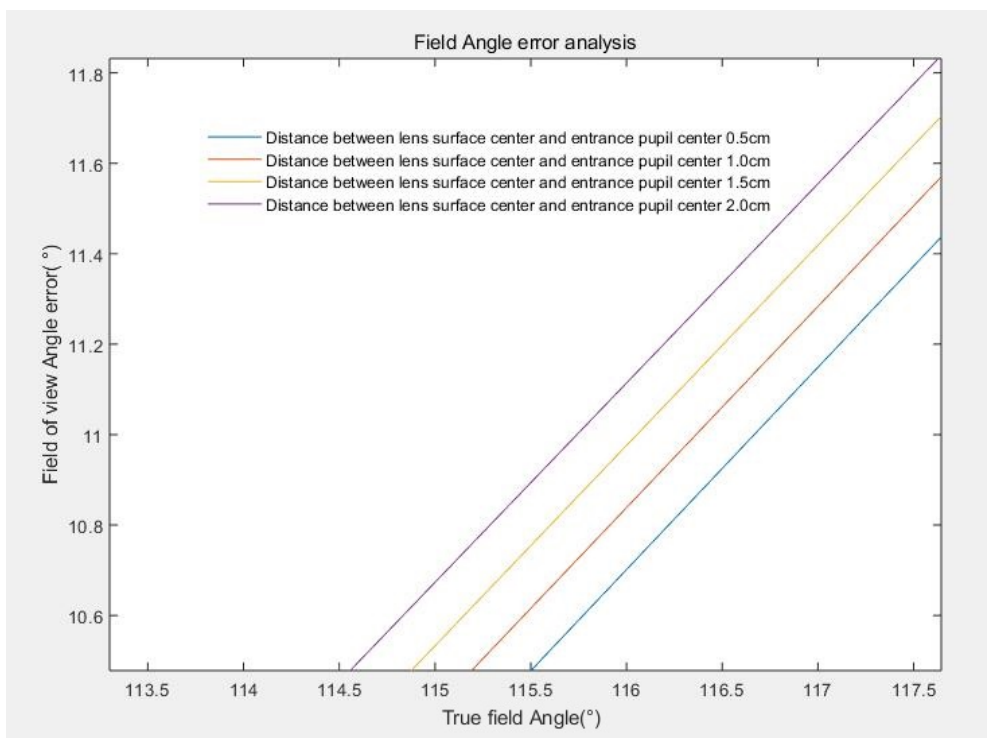

(a) 


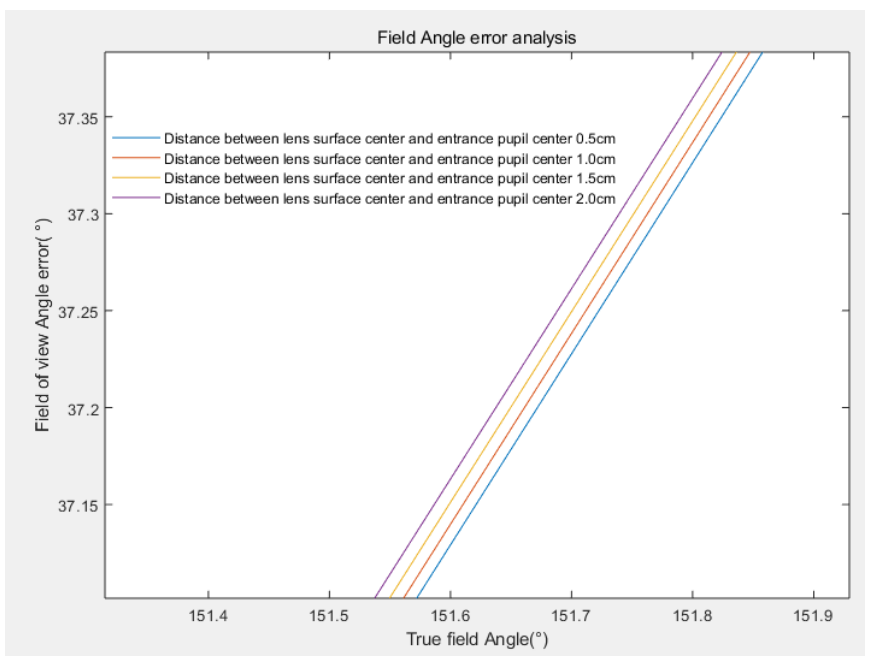

(b)

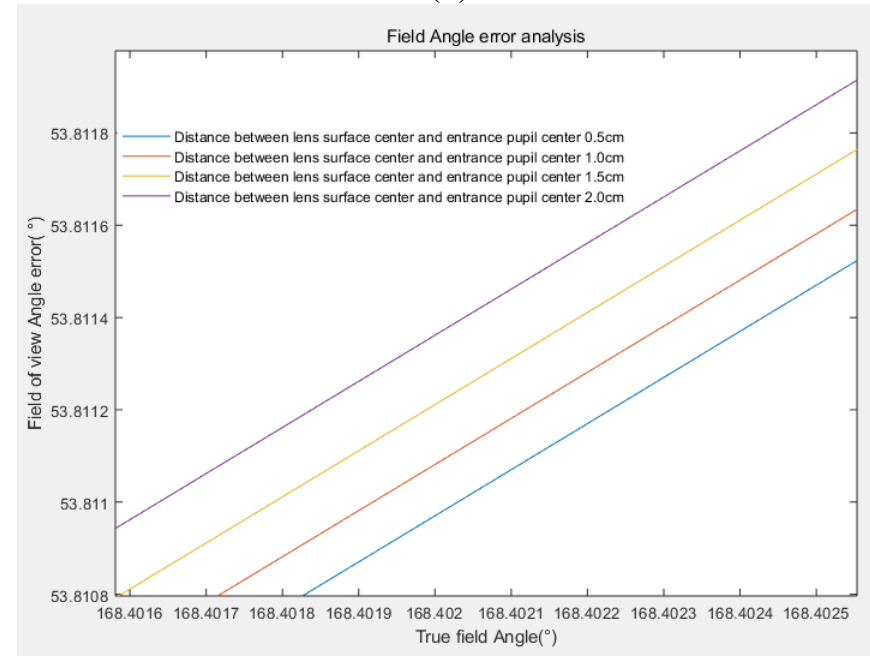

(c)

Fig. 6 (a), (b) , (c) Variation chart of angle error of FOV with real FOV angle.

\section{Wide range horizontal field of view Angle measurement method and realization device}

Aiming at the problem of measuring field angle error caused by the distance $\mathrm{HO}$ between the front surface of the camera lens and the center of entry pupil, Li Kun ${ }^{[1]}$ et al. put forward calibration method that can effectively avoid the error, but for laboratory measurement of wide range of horizontal viewing angle, it is not applicable. This paper proposes a wide range of horizontal viewing angle test method, the device is supported by the camera movement, imaging target test board and control computer, as shown in figure.7. Among them, the control computer is connected with the camera support mobile platform to control the movement of the support mobile platform. The imaging target test board is provided with a left and right symmetrical scale ruler centered on the origin. The initial position of the camera to be tested is adjusted so that its center is aligned with the center of the scale graph of the target test board. Through the control computer to control the 
movement of the supporting mobile platform, the camera's field of view boundary and scale graph center width D2, D1 and the distance $\Delta \mathrm{L}$ between a and b were recorded respectively when the camera was in position a and $b$. The field angle of the camera to be tested can be calculated according to the following formula (6) :

$$
\theta=2 \times \arctan \left(\frac{D_{2}-D_{1}}{\Delta L}\right)
$$

Further, the target test board and its scale graphics respectively have extensions perpendicular to the plane at both ends. When the field angle of the camera to be tested is very large, the field of view boundary of the camera to be tested is recorded at position A, D3 of the scale extension segment, and the position of the camera to be tested is moved to B. Record the width D1 of the camera's field of view boundary and the scale graph center, and record the width D0 of the scale graph center and the scale boundary. Read the extended scale to obtain the overall field of view angle value of the equipment to be tested. The field angle can be calculated according to formula (7) as below:

$$
\theta=2 \times \arctan \left(\frac{D_{1}-D_{0}}{D_{3}-\Delta L}\right)
$$

\section{Conclusion}

In this paper, it is concluded that the wide range of field angle can reduce the experimental error.by analyzing the principle of field angle error and using the method of laboratory simulation, In addition, a wide range of horizontal field of view angle measurement method is proposed, in which the camera is shifted on the support mobile platform to image the imaging target test board, and then the field angle of the camera is calculated by reading the scale value on the imaging ta rget test board. The method has advantages of simple operation, good repeatability, reduced testing time and improved testing efficiency. It is suitable for field angle testing of common range cameras and field calibration of cameras with large range of field angle (wide-angle camera or fisheye camera, etc.).

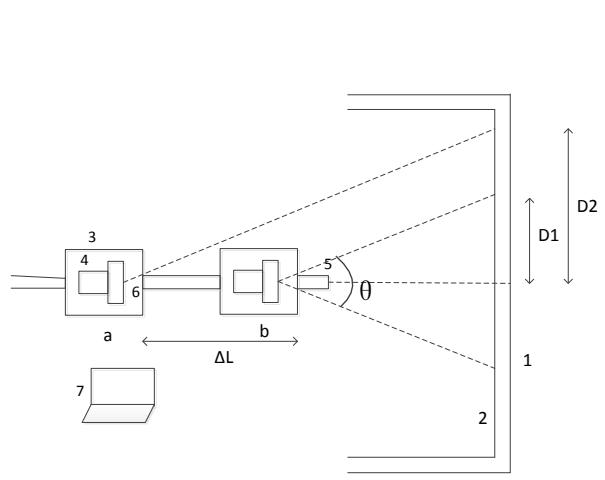

(a)

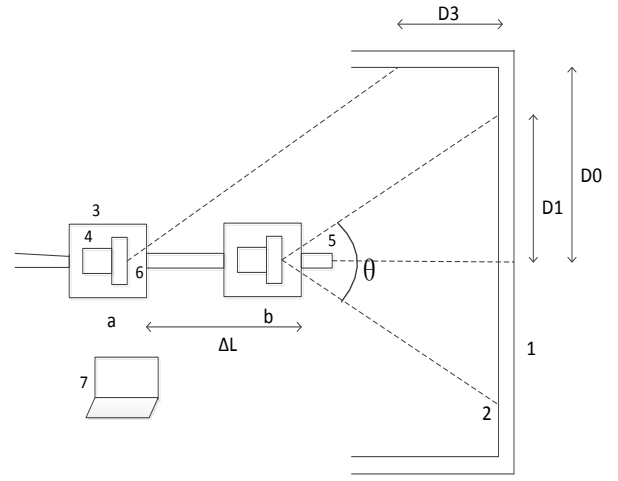

(b)

Fig. 7. (a), (b) Measurement of wide range horizontal field of view.

\section{References}

1. Lili Jiang, Dehua Liu. Transmission film test card and application method for camera testing [P], CN100541325, 2009. 
2. Li Yuan. Measurement on Camera Vision [J]. Science and technology information, 2011 (32): 38-38.

3. Kun Li, Yongquan Chen, Jianke Zhao. Calibration method and device of camera vision [P], CN201310626307, 2013.

4. Zhe Li, Weidong Fan, Yuan Li. Research on the Optical Parameter Testing Technique of the Semiconductor Laser Fuze [J]. GUIDANCE \& FUZE, 2013, 34(2): 13-18.

5. Zuojun Tan, Haiqing Chen, Jingran Kang. Research and Test of Receiver Module in Laser Fuze [J]. 2009, 38(11): 2742-2746.

6. Rupeng Feng, Deping Zhong, Yongbo Fa. Data processing on shooting and receiving view angle measurement of laser guidance with three-axis test table [J]. Journal of Chinese Inertial Technology, 2004, 12 (4): 65-69.

7. Bowen Yang, Liyan Zhang, Nan Ye. Camera Calibration Technique of Wide-Area Vision Measurement [J]. ACTA OPTICA SINICA, 2013, 32(9):091500.

8. Wukang Lin. Research on Camera Calibration Method Of Wide Field[D]. Harbin: Harbin Institute of Technology, 2011:13-42.

9. En Peng, Li Ling. Camera calibration using one-dimensional information and its application in both controlled and uncontrolled environments[J]. Pattern Recognition, 2010, 43(S0031-3203): 1100-1198.

10. Ting Gao. Research and Application of the Short Focus Length Camera Calibration in Wide Field[D]. Xi 'an: Xidian University, 2014: 15-24.

11. Weimin Li, Qiaoyun Yu, Chao Liu. Calibration Method with Separation Pattern of a Single-Camera Based on Difference Coordinates [J]. Acta Optica Sinica, 2006, 26(5):51-55.

12. Weimin Li, Hao Meng, Jianping Wang. Wide view calibration of single cameras [J]. Journal of University of Science and Technology of China. 2007, 37(6):627-630.

13. Dong Ye, Qiaoyu Xu, Rensheng Che. Camera calibration technique for vision measurement system [J]. Optics and Precision Engineering. 2006, 14(5):883-890.

14. Dong Ye, Changying Liu, Rensheng Che. Dummy solid camera calibration technology using genetic algorithm [J]. Optics and Precision Engineering. 2006, 14(3):485-489.

15. Junhua Sun, Zhen Liu, Guangjun Zhang. Camera Calibration Based on Flexible 3D Target [J]. Acta Optica Sinica. 2009, 29(12):3433-3439.

16. Yan An, Yiyun Xu, Keyan Dong. Detection of FOV for portable laser communication systems [J]. Laser Technology. 2019, 43(1):15-18.

17. Keyan Dong, Yiyun Xu, Yan An. A portable measuring device for receiving field angle of view of laser communication [P]. CN201810185699.1,2019. 